\title{
Cardiopulmonary Bypass Induced Hematological Changes in Patients Undergoing Cardiac Surgery
}

\author{
Md. Aftabuddin ${ }^{1}$, Nivesh Rajbhandhari², Md. Zillur Rahman ${ }^{3}$, Nadira Islam ${ }^{4}$, Omar Sadeuque Khan $^{5}$
}

\begin{abstract}
:
Cardiopulmonary bypass (CPB) assisted open heart surgery have become a frequent surgical procedures in almost all major heart surgeries. However,growing evidence indicates that anemia, coagulation derangements, platelet dysfunction and inflammatory responses are common in patients undergoing CPB aided cardiac surgery. The aim of the present study was to characterize CPB induced hematologic changes in patients undergoing cardiac surgery. A total of sixty three patients undergoing elective cardiac surgery in the department of Cardiac Surgery, BSMMU, Dhaka, Bangladesh, were prospectively randomized into the present study: 33 of which underwent CPB aided cardiac surgery (CPB-aided) and the rest 30 patients underwent cardiac surgery without CPB (CPB non-aided). Complete blood count together with $\mathrm{Hb} \%$, prothrombin time (PT) and International normalized ratio (INR) was determined preoperatively and on $1^{\text {st }}, 3^{\text {rd }}$ and $7^{\text {th }}$ postoperative days (POD). The TC of WBC was significantly higher in CPB
\end{abstract}

aided patients than that of CPB non-aided patients on $1^{\text {st }}$ POD. When comparison was done, total count of RBC, platelets \& $\mathrm{Hb} \%$ were significantly lower in CPB aided patients compared to patients underwent cardiac surgery without CPB on the $7^{\text {th }}$ POD. Additionally, PT and INR were higher in CPB aided cardiac patients compared to that of CPB non-aided patients on the 7th POD. The lower levels of $\mathrm{Hb} \%$ and $\mathrm{TC}$ of RBC following CPB-aided cardiac surgery may be due to excessive hemolysis because of mechanical force during extra-corporal circulation. The observed significant leucocytosis in the present CPB aided cardiac surgery patients on $1^{\text {st }} P O D$ may support the notion that CPB induces a systemic inflammatory response following cardiac surgery. Further studies are required to evaluate the mechanisms of CPB- induced hematological changes following major cardiac surgery.

Key words: Cardiopulmonary bypass, Hemoglobin, Prothrombin time.
Introduction:

Cardiopulmonary bypass (CPB) is a technique that temporarily takes over the function of heart and lungs during surgery, maintaining the circulation of blood and the oxygen content of the body ${ }^{1}$. This technique has been a revolutionary innovation in medical science that has allowed cardiac surgeons to perform various complicated open heart surgeries, such as valve replacement, repair

1. Professor, Department of Cardiac surgery, Bangabandhu Sheikh Mujib Medical University, Dhaka.

2. Resident, Teaching Hospital, Kathmandu, Nepal.

3. Associate Professor and Head, Department of Biochemistry, Khulna Medical Collage, Khulna.

4. Professor, Department of Physiology, Delta Medical Collage, Dhaka.

5. Medical Officer, Department of Cardiac surgery, Bangabandhu Sheikh Mujib Medical University, Dhaka. of congenital anomalies, coronary bypass surgery, and repair of some large aneurysm, pulmonaryembolectomy and heart transplantation, with great convenience ${ }^{1}$. However, it has been observed that patients undergoing cardiac surgery with CPB are at increased risk for excessive perioperative blood loss, and postoperative anemia, jaundice and infection ${ }^{2}$, though extensive efforts are routinely done to minimize damaging factors.

During CPB, forces on the formed elements of the blood induced through mechanical stress and other environmental factors result in different degrees of damage to these cells. Although efforts are made to minimize RBC damage during $\mathrm{CPB}$, the potential consequences of excessive hemolysis like decreased $\mathrm{TC}$ of $\mathrm{RBC}$ and $\mathrm{Hb} \%$, following $\mathrm{CPB}$-aided cardiac 
surgery cannot be avoided ${ }^{2}$. The patho-physioloigcal aggregation of RBC is changed during CPB mainly due to presence of priming solution causing hemodilution and drop in blood viscosity. Along with hypoxia and inflammatory response surgical manipulation also plays an important role in altering RBC aggregability ${ }^{3}$.

It has also been suggested that CPB-aided cardiac surgery is associated with low count of platelets and lower level of coagulation factors ${ }^{2}$. Researchers have suggested that CPB induced hemodilution may, in part, account for the decreases of coagulation factors and platelets count. It has been found that extracorporeal circulation triggers massive defense reaction that involves endothelial cell and white blood cells proliferation ${ }^{3}$. Various studies have been suggested that the use of CPB is associated with a generalized hemorrhagic defect ${ }^{4}, 5$; and platelet dysfunction and decreased platelet number have been considered as the major contributing factors ${ }^{6}$. An elevated prothrombin time (PT) is considered as a potential threat to increased intra operative and postoperative bleeding and hence necessitates close monitoring of PT both before and after CPB-aided cardiac surgery.

Cardiopulmonary bypass induced changes in hematological and hemorrhagic factors are yet not conclusive; especially such study has not been done on cardiac patients in Bangladesh. Therefore, the present study was designed to evaluate the hematological changes together with PT and International normalized ratio (INR) in patients undergoing cardiac surgery using CPB.

\section{Methods and materials:}

The study was carried out in the department of cardiac surgery, BSMMU, Dhaka, Bangladesh, July 2008 to June 2013. The ethical review committee of the university approved the study protocol. A total of 63 patients aged 20 to 55 of either sex, who underwent various elective cardiac surgeries using CPB, were randomly selected during this study period that satisfied the inclusion criteria: left ventricular ejection fraction (EF) more than 35\%, patient in New York heart association (NYHA) class I to III. Patients, who had cyanotic heart diseases, altered liver and renal function, emergency cardiac surgery, and redo cardiac surgery having history of recent myocardial infarction (MI) within 6 weeks and where intra-aortic balloon pump (IABP) applied was excluded.Informed written consent was taken from each patient after explaining the nature, purpose and importance of the study. Out of 63 selected patients, CPB was used in 33 patients (CPB-aided group) and in the rest 30 patients; $\mathrm{CPB}$ was not used (CPB non-aided group)

For hematological and hemorrhagic parameters, complete blood cell count, hemoglobin concentration, PT, and international normalized ratio (INR) were measured using standard laboratory techniquesbefore operation (preoperative) and after $1^{\text {st }}, 3^{\text {rd }}$, and $7^{\text {th }}$ days of operation $\left(1^{\text {st }}, 3^{\text {rd }}, 7^{\text {th }} \mathrm{POD}\right)$. All information regarding patients, such as type of operation, age, sex, clinical variables, preoperative medications, intra-operative blood loss and transfusion or any intra-operative complications, was recorded in a pre-designed data sheet. Post-operative blood loss and transfusion were also recorded. All data were collected, complied and then analyzed using SPSS for windows software (version 16), Student's t test and chi- square test done accordingly. P value $\leq 0.05$ was considered significant.

\section{Results:}

The mean TC of RBC and $\mathrm{Hb} \%$ were progressively decreased in CPB-aided cardiac patients following operation compared to that their pre-operative values. Although similar trend was observed in patients operated without $\mathrm{CPB}$, the differences were not significant. On the other hand, the mean TC of WBC was significantly increased on $1^{\text {st }} P O D$ in patients underwent cardiac surgery with CPB when compared to that of their preoperative total count. However, the TC of WBC gradually decreased on $3^{\text {rd }}$ and $7^{\text {th }}$ POD. Again, the platelet count declined on $1^{\text {st }}$ and $3^{\text {rd }}$ POD from preoperative period in patients underwent CPB-aided cardiac surgery, though platelet count increased to almost its preoperative level on $7^{\text {th }}$ POD. PT and international normalized ratio (INR) were progressively increased from $1^{\text {st }} P O D$ to $7^{\text {th }}$ POD in CPB-aided patients.

The mean preoperative values of different parameters were almost similar in both groups (CPB-aided vs CPB non-aided group); the differences were insignificant (Table No. 1). However, the mean TC of RBC and $\mathrm{Hb} \%$ were lower in patients underwent CPB-aided cardiac surgery compared to that to patients underwent cardiac surgery without CPB on $1^{\text {st }}, 3^{\text {rd }}$ and $7^{\text {th }}$ POD (Table No. 1). The TC of WBC was significantly higher in CPB-aided patients on $1^{\text {st }}$ POD in comparison to that of CPB nonaided patients. However, the differences were not significant on $3^{\text {rd }}$ and $7^{\text {th }}$ POD between two groups. PT and INR were almost similar when compared between two groups on $1^{\text {st }}$ and $3^{\text {rd }}$ POD (Table No. 2). However, on the $7^{\text {th }}$ POD, the mean PT and INR weresignificantly higher in patients underwent CPB-aided cardiac patients compared to that of non-aided group. 
Table I

Mean ( $(\mathrm{SD})$ blood cell counts and $\mathrm{Hb} \%$ in patients undergoing cardiac surgery with and without CPB

\begin{tabular}{|c|c|c|c|}
\hline Parameter & Time & $\begin{array}{l}\text { CPB-aided Patients } \\
(n=33)\end{array}$ & $\begin{array}{l}\text { CPB non-aided Patients } \\
\qquad(\mathrm{n}=30)\end{array}$ \\
\hline \multirow[t]{4}{*}{ TC of RBC (million/cu-mm) } & Preoperative & $4.36 \pm 4.4$ & $4.46 \pm 0.45 \mathrm{NS}$ \\
\hline & $1^{\text {st }} \mathrm{POD}$ & $4.05 \pm 0.38$ & $4.26 \pm 0.66 \mathrm{NS}$ \\
\hline & $3^{\text {rd }}$ POD & $3.95 \pm 0.5$ & $4.05 \pm 0.46 \mathrm{Ns}$ \\
\hline & $7^{\text {th }}$ POD & $3.89 \pm 0.48$ & $4.15 \pm 0.5 S$ \\
\hline \multirow[t]{4}{*}{$\mathrm{Hb} \%(\mathrm{gm} \mathrm{\% )}$} & Preoperative & $12.2 \pm 1.4$ & $12.4 \pm 1.5 \mathrm{NS}$ \\
\hline & $1^{\text {st }} \mathrm{POD}$ & $11.1 \pm 1.2$ & $11.7 \pm 1.7 \mathrm{NS}$ \\
\hline & $3^{\text {rd }}$ POD & $11.0 \pm 1.3$ & $11.2 \pm 1.2 \mathrm{NS}$ \\
\hline & $7^{\text {th }} P O D$ & $10.6 \pm 1.1$ & $11.6 \pm 1.3 S$ \\
\hline \multirow[t]{4}{*}{ TC of WBC(cells/cu-mm) } & Preoperative & $9070 \pm 206$ & $9087 \pm 192 N S$ \\
\hline & $1^{\text {st }} \mathrm{POD}$ & $17139 \pm 563$ & $14774 \pm 318 N S$ \\
\hline & $3^{\text {rd }}$ POD & $11512 \pm 475$ & $11086 \pm 293 N S$ \\
\hline & $7^{\text {th }}$ POD & $11988 \pm 301$ & $11086 \pm 293 S$ \\
\hline \multirow[t]{4}{*}{ Platelets count (lacs/cu-mm) } & Preoperative & $2.28 \pm 0.51$ & $2.45 \pm 0.48 \mathrm{NS}$ \\
\hline & $1^{\text {st }} \mathrm{POD}$ & $1.93 \pm 0.45$ & $2.05 \pm 0.38 \mathrm{NS}$ \\
\hline & $3^{\text {rd }}$ POD & $1.81 \pm 0.38$ & $2.0 \pm 0.63 \mathrm{NS}$ \\
\hline & $7^{\text {th }}$ POD & $2.23 \pm 0.41$ & $2.53 \pm 0.72 \mathrm{~S}$ \\
\hline
\end{tabular}

Unpaired' $t$ ' test was done for teat of significance; $S=$ significant; ns =not significant; $\mathrm{n}=$ number of patients

Table-II

Mean $( \pm S D)$ prothrombin time $(P T)$ and international normalized ratio (INR) in patients undergoing cardiac surgery with and without $C P B$

\begin{tabular}{lccc}
\hline Parameter & Time & $\begin{array}{c}\text { CPB-aided Patients } \\
(\mathrm{n}=33)\end{array}$ & $\begin{array}{c}\text { CPB non-aided Patients } \\
(\mathrm{n}=30)\end{array}$ \\
\hline PT $(\mathrm{sec})$ & Preoperative & $13.5 \pm 2.5$ & $13.7 \pm 1.7 \mathrm{NS}$ \\
& $1^{\text {st } P O D}$ & $16.7 \pm 3.1$ & $17.2 \pm 2.9 \mathrm{NS}$ \\
& $3^{\text {rd POD }}$ & $16.5 \pm 5.2$ & $16.0 \pm 3.0 \mathrm{NS}$ \\
INR & $7^{\text {th POD }}$ & $19.1 \pm 8.3$ & $15.7 \pm 2.8 \mathrm{~S}$ \\
& Preoperative & $1.1 \pm 0.2$ & $1.1 \pm 0.1 \mathrm{NS}$ \\
& $1^{\text {st POD }}$ & $1.4 \pm 0.3$ & $1.4 \pm 0.3 \mathrm{NS}$ \\
& $3^{\text {rd POD }}$ & $1.4 \pm 0.4$ & $1.3 \pm 0.3 \mathrm{NS}$ \\
\hline
\end{tabular}

Unpaired ' $\mathrm{t}$ ' test was done for test of significance; $\mathrm{NS}=$ not significant, $\mathrm{S}=$ significant $\mathrm{n}=$ number of patients

\section{Discussion :}

In the present study, TC of RBC and $\mathrm{Hb} \%$ were significantly lower in patients underwent elective open heart surgery using CPB following operation. In consistent with the present study, Salama et al. also observed a decrease in RBC count during the first 3 to 4 days following operation ${ }^{7}$. In CPB-aided cardiac surgery, hemodilution cannot be avoided as from many points of view, hemodilution throughout CPB is considered beneficial for outcome of surgery. Hemodilution induced anemia is common in CPB-aided cardiac surgery. Apart from that, it has been suggested that mechanical forces during extracorporal circulation can cause complete destruction of the RBC, immediate or delayed. Additionally, CPB is also known to cause significant changes in the mechanical properties of $\mathrm{RBC}^{8}$, such as decreasing their deformability and surface charge and increasing their fragility and aggregability ${ }^{9}$. Some studies have indicated 
sublethal damage by CPB instead of complete destruction, where RBC exposed to certain uniform shear stresses for prolonged time show significant changes in their deformability without lysis ${ }^{10}$. One study showed that $15 \%$ of the RBC subjected to CPB were irreversibly damaged and removed during the 24 hour post-infusion period $^{11}$. Therefore, beside hemodilution anemia, the observed lower TC of RBC and $\mathrm{Hb} \%$ in patients following CPB-aided cardiac surgery may be, at least partly, due to excessive hemolysis because of mechanical trauma from CPB procedure.

In our study population, the TC of WBC was significantly higher in patients underwent cardiac surgery using CPB in their postoperative days. In contrast, other researchers have observed that during CPB an initial mild leukopenia develops which soon returns to baseline ${ }^{12}$. However, in support of our findings, it has been found that leukocytes count often increase to a peak of 24000 cells $\mathrm{ml}^{-3}$ at 24 to 48 hours post -operatively ${ }^{13}$. Although the precise mechanism of leucocytosis after CPB-aided cardiac surgery is unknown, it has been suggested that granulocyte colony stimulating factor (G-CSF) plays an important role as the mediator of granulocytes after cardiac surgery with CPB. Additionally, CPB is known to induce a systemic inflammatory response by triggering the production and release of a multitude of inflammatory mediators. Thus, the presence of higher number of WBC in our patients who underwent CPB-aided cardiac surgery within $24 \mathrm{hrs}$ of surgery may indicate an activation of G-CSF and inflammatory mediators on exposure of blood to CPB circuit.

Although after operation, the platelet count was lower in our CPB-aided patients compared to that of non-aided patients, it returned almost to its preoperative count on $7^{\text {th }}$ POD. In contrary to our result, it was observed that platelets declined about $50 \%$ of preoperative levels soon after starting $\mathrm{CPB}^{6}$ and several days required to correct the platelet count ${ }^{6}$.Although platelet count remains within normal range following bypass, progressive lowering of number of platelets in our CPB-aided patients may be a sign of progressive loss of platelet function, influencing the aggregation of platelet to adenosine diphosphate (ADP). Platelet dysfunction appears to be dependent on contact of platelet with synthetic surfaces of the extra corporeal oxygenator and hypothermia associated with bypass.

Earlier studies have found that the PT and IN Rare usually normal following discontinuation of CPB and protamine administration ${ }^{16}$. It has been suggested that shortly after starting of CPB, plasma concentration of prothombin reduces, and such reduction is primarily due to hemodilution ${ }^{12,13}$. So, the observed significantly prolonged PT in our patients who underwent CPB-aided cardiac surgery during postoperative period may be due to CPB procedure induced hemodilution.

\section{Conclusion:}

This study observed significant changes in various hematological and hemorrhagic variables such as altered blood cells count, hemoglobin level, PT and INR in patients underwent cardiac surgery with CPB during early postoperative days in comparison to patients undergoing cardiac surgery without CPB. Further studies are required to evaluate the mechanisms of CPB-induced hematological and hemorrhagic changes following major cardiac surgery.

\section{References:}

1. Copper JR,Glesecke NM, Cardiopulmonary bypass: Principle and practice. $2^{\text {nd }}$ ed,Philadelphia: Lippincott William \&Wilkins, 2000:186-96.

2. De Somer F. Optimisation of the perfusion circuit and its possible impact on the inflammatory response. J Extra CorporTecnal. 2007; 39:285-8.

3. Bhujle R, Li-J, shastri P, et al. Influence of cardiopulmonary bypass on platelet and Neutrophil accumulation in internal organ. ASAIO J. 1997 SepOct;43(5):M739-44.

4. Moraria AM, Gu YJ, Huet RCGH, Siamons WA, Rakhorst G, Oeveren WV. Red blood cell aggregation during cardiopulmonary bypass: a pathogenic cofactor in endothelial cell activations? Eur J. Cardiothoracic Surg. 2004; 26: 939-46.

5. Martine Z, Vaya A, Server R, Gilsan ZA, Aznar J. alterations in erythrocyte aggregability in diabetes: the influence of plasmatic fibrinogen and phospholipid of the RBC cell membrane. Clin Hemorheol Microcircal 1998; 18:253-8.

6. Richard C, Woodman, Laurence AH. Bleeding complications associate with Cardiopulmonary bypass. Blood 1990; 76:1680-97.

7. Salama A, Hugo F, heinrich D, et al. Deposition of terminal $C_{5}$ b-9 complement complexes during cardiopulmonary bypass. N Engl J Med 1988; 318: 408-20.

8. Vercaemst L. Hemolysis in cardio surgery patients undergoing Cardiopulmonary bypass: A review in search of a treatment algorithm. JECT 2008, 40:257-67. 
9. Kameneva M, Hemorheology of mechanical blood damage. Presented at:The $3^{\text {rd }}$ international conference on pediatric mechanical circulatory support and pediatric CPB, Hershey,PA. $17-19^{\text {th }}$ May 2007.

10. Watanabee N, Arakawa Y, Atsushi S, et al. Deformability of human red blood cells exposed to a uniform shear stress as measured by a cyclically reversing flow generator. Physiol Meas. 2007, 28: 531-45.

11. Valeri R, McGregor H, Rango G, Healey N, Fonger J, Khuri S. Effects of centrifugal and roller pumps on survival of autologous red cells in cardiopulmonary bypass surgery. Perfusion 2006; 21: 291.

12. Patarroyo M, Makgoba MW, leukocyte adhesionto cells in immune and inflammatory response. Lancet 1989; 2: 1139-51.
13. Riegel W, spillner G, Schlosser V, Horl WH. plasma levels of main granulocyte componentsduring cardiopulmonary bypass. J Thorac Cardiovasc Surg 1988; 95(6): 1014-9.

14. Harker LA, Malpass TW, Branson HE, Hessel EA 2nd, Slichter SJ. Mechanism of abnormal bleeding in patient going under cardiopulmonary bypass: acquired transient platelet dysfunction associated with sedative a granule release. Blood 1980; 56: 824-35.

15. Brody JI, Pickering NJ, Fink GB . Concentration of factor VIII- related antigen and factor XIII during open heart surgery. Transfusion 1986; 26 :478-89.

16. Milam JD, Austin SF, Martin RF, Keats AS, Cooley DA. Alteration of coagulation and selected clinical chemistry parameters in patients undergoing open heart surgery without transfusion. Am J Clin Pathol 1981; 76: 155-62. 